\title{
On the line-symmetry of self-motions of linear pentapods
}

\author{
Georg Nawratil
}

\begin{abstract}
We show that all self-motions of pentapods with linear platform of Type 1 and Type 2 can be generated by line-symmetric motions. Thus this paper closes a gap between the more than 100 year old works of Duporcq and Borel and the extensive study of line-symmetric motions done by Krames in the 1930's. As a consequence we also get a new solution set for the Borel Bricard problem. Moreover we discuss the reality of self-motions and give a sufficient condition for the design of linear pentapods of Type 1 and Type 2, which have a self-motion free workspace.
\end{abstract}

Key words: Linear Pentapod, Self-motion, Line-symmetric motion, Borel-Bricard problem

\section{Introduction}

The geometry of a linear pentapod is given by the five base anchor points $\mathrm{M}_{i}$ in the fixed system $\Sigma_{0}$ and by the five collinear platform anchor points $\mathrm{m}_{i}$ in the moving system $\Sigma$ (for $i=1, \ldots, 5$ ). Each pair $\left(\mathrm{M}_{i}, \mathrm{~m}_{i}\right)$ of corresponding anchor points is connected by a SPS-leg, where only the prismatic joint is active.

If the geometry of the manipulator is given as well as the lengths $R_{i}$ of the five pairwise distinct legs, a linear pentapod has generically mobility 1 , which corresponds to the rotation about the carrier line $\mathrm{p}$ of the five platform anchor points. As this rotational motion is irrelevant for applications with axial symmetry (e.g. 5axis milling, spot-welding, laser or water-jet engraving/cutting, spray-based painting, etc.), these mechanisms are of great practical interest. Nevertheless configurations should be avoided where the manipulator gains an additional uncontrollable mobility, which is referred as self-motion.

Georg Nawratil

Institute of Discrete Mathematics and Geometry, Vienna University of Technology, Austria, e-mail: nawratilegeometrie.tuwien.ac.at 


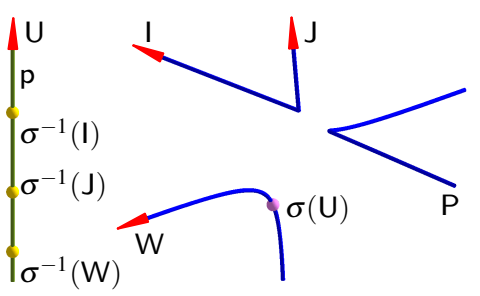

(a)

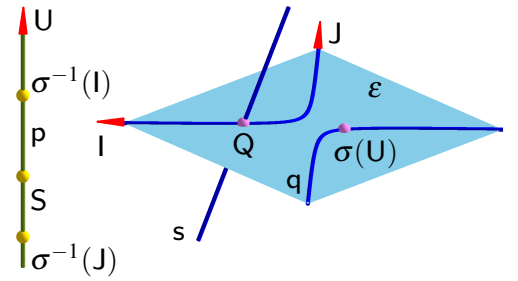

(b)

Fig. 1 Projective sketch of linear pentapods of (a) Type 1 and (b) Type 2, respectively, with selfmotions.

\subsection{Review on self-motions of linear pentapods}

The self-motions of linear pentapods represent interesting solutions to the still unsolved problem posed by the French Academy of Science for the Prix Vaillant of the year 1904, which is also known as Borel-Bricard problem (cf. [2, 3, 4]) and reads as follows: "Determine and study all displacements of a rigid body in which distinct points of the body move on spherical paths."

For the special case of five collinear points the Borel-Bricard problem was studied by Darboux [6, page 222], Mannheim [7, pages 180ff] and Duporcq [8] (see also Bricard [3, Chapter III]). A contemporary and accurate reexamination of these old results, which also takes the coincidence of platform anchor points into account, was done in [1] yielding a full classification of linear pentapods with self-motions.

Beside the architecturally singular linear pentapods [1, Corollary 1] and some trivial cases with pure rotational self-motions [1, Designs $\alpha, \beta, \gamma]$ or pure translational ones [1, Theorem 1] there only remain the following three designs:

Under a self-motion each point of the line $\mathrm{p}$ has a spherical (or planar) trajectory. The locus of the corresponding sphere centers is a space curve $\mathrm{P}$ of degree 3 , where the mapping from $p$ to $P$ is named $\sigma$. $P$ intersects the ideal plane in one real point $W$ and two conjugate complex ideal points, where the latter ones are the cyclic points I and $\mathrm{J}$ of a plane orthogonal to the direction of W. $\mathrm{P}$ is therefore a so-called straight cubic circle. The following subcases can be distinguished:

- $\mathrm{P}$ is irreducible:

- $\sigma$ maps the ideal point $U$ of $\mathrm{p}$ to $\mathrm{W}$ (Type 5 according to [1]).

- $\sigma$ maps $U$ to a finite point of $\mathrm{P}$ (Type 1 according to [1]; see Fig. 117).

- P splits up into a circle $q$ and a line $s$, which is orthogonal to the carrier plane $\varepsilon$ of $q$ and intersects $q$ in a point $Q$. There is a bijection $\sigma$ between $p \backslash\{S\}$ and $q \backslash\{Q\}$; moreover the finite point $S$ is mapped to s. As a consequence $\sigma$ maps $U$ to a point on the circle different from $Q$ (Type 2 according to [1]; see Fig. 1p). 


\subsection{Basics on line-symmetric motions}

Krames (e.g. [5, 11]) studied special one-parametric motions (Symmetrische Schrotung in German), which are obtained by reflecting the moving system $\Sigma$ in the generators of a ruled surface of the fixed system $\Sigma_{0}$, which is the so called basic surface. These so-called line-symmetric motions were also studied by Bottema and Roth [9. $\S 7$ of Chapter 9], who gave an intuitive algebraic characterization in terms of Study parameters $\left(e_{0}: e_{1}: e_{2}: e_{3}: f_{0}: f_{1}: f_{2}: f_{3}\right)$, which are shortly repeated next.

All real points of the Study parameter space $P^{7}$ (7-dimensional projective space), which are located on the so-called Study quadric $\Psi: \sum_{i=0}^{3} e_{i} f_{i}=0$, correspond to an Euclidean displacement with exception of the 3-dimensional subspace $e_{0}=e_{1}=$ $e_{2}=e_{3}=0$, as its points cannot fulfill the condition $N \neq 0$ with $N:=e_{0}^{2}+e_{1}^{2}+$ $e_{2}^{2}+e_{3}^{2}$. The translation vector $\mathbf{s}:=\left(s_{1}, s_{2}, s_{3}\right)^{T}$ and the rotation matrix $\mathbf{R}$ of the corresponding Euclidean displacement $\mathbf{m}_{i} \mapsto \mathbf{R m}_{i}+\mathbf{s}$ are given for $N=1$ by:

$$
\begin{aligned}
& s_{1}=-2\left(e_{0} f_{1}-e_{1} f_{0}+e_{2} f_{3}-e_{3} f_{2}\right), \quad s_{2}=-2\left(e_{0} f_{2}-e_{2} f_{0}+e_{3} f_{1}-e_{1} f_{3}\right), \\
& s_{3}=-2\left(e_{0} f_{3}-e_{3} f_{0}+e_{1} f_{2}-e_{2} f_{1}\right), \\
& \mathbf{R}=\left(\begin{array}{lll}
r_{11} & r_{12} & r_{13} \\
r_{21} & r_{22} & r_{23} \\
r_{31} & r_{32} & r_{33}
\end{array}\right)=\left(\begin{array}{ccc}
e_{0}^{2}+e_{1}^{2}-e_{2}^{2}-e_{3}^{2} & 2\left(e_{1} e_{2}-e_{0} e_{3}\right) & 2\left(e_{1} e_{3}+e_{0} e_{2}\right) \\
2\left(e_{1} e_{2}+e_{0} e_{3}\right) & e_{0}^{2}-e_{1}^{2}+e_{2}^{2}-e_{3}^{2} & 2\left(e_{2} e_{3}-e_{0} e_{1}\right) \\
2\left(e_{1} e_{3}-e_{0} e_{2}\right) & 2\left(e_{2} e_{3}+e_{0} e_{1}\right) & e_{0}^{2}-e_{1}^{2}-e_{2}^{2}+e_{3}^{2}
\end{array}\right) .
\end{aligned}
$$

There always exists a moving frame (in dependence of a given fixed frame) in a way that $e_{0}=f_{0}=0$ holds for a line-symmetric motion. Then $\left(e_{1}: e_{2}: e_{3}: f_{1}\right.$ : $\left.f_{2}: f_{3}\right)$ are the Plücker coordinates (according to the convention used in [9]) of the generators of the basic surface with respect to the fixed frame.

\subsection{Line-symmetric self-motions of linear pentapods}

It is well known (cf. [8, $\S 15],[3, \S 12]$ ) that the self-motions of Type 5 are obtained by restricting the Borel-Bricard motions 1 (also known as BB-I motions) to a line. Note that this special case was also discussed in detail by Krames [5, Section 5], who also pointed out the line-symmetry of BB-I motions.

Beside these BB-I motions, there also exist line-symmetric motions (so-called BB-II motions), where all points of a hyperboloid carrying two reguli of lines have spherical trajectories. It is known (cf. [10, page 24] and [11, page 188]) that the corresponding sphere centers of lines, belonging to one regulus form irreducible straight cubic circles, which imply examples of self-motions of Type 1 . Note that

\footnotetext{
${ }^{1}$ These are the only non-trivial motions where all points of the moving space have spherical trajectories (cf. [3] Chapter VI]).

2 The corresponding sphere centers of lines belonging to the other regulus are again located on a line (cf. [10. page 24]), which imply architecturally singular designs of linear pentapods.
} 
there also exist degenerated cases where the hyperboloid splits up into two orthogonal planes, which contain examples of self-motions of Type 2 .

A simple count of free parameters shows that not all self-motions of Type 1 (5parametric se ${ }^{3}$ of motions where all points of a line have spherical paths) can be generated by BBM-II motions (which produce only a 4-parametric se 4 ). The same argumentation holds for Type 2 self-motions and the mentioned degenerated case.

As a consequence the question arise whether all self-motions of linear pentapods of Type 1 and Type 2 can be generated by line-symmetric motions. If this is the case we can apply a construction proposed by Krames [5, page 416], which is discussed in Section 4, yielding new solutions to the Borel-Bricard problem.

\section{One the line-symmetry of Type 1 and Type 2 self-motions}

For our calculations we do not select arbitrary pairs $\left(m_{i}, M_{i}\right)$ of $\mathrm{p}$ and $\mathrm{P}$, which are in correspondence with respect to $\sigma\left(\Leftrightarrow \sigma\left(\mathrm{m}_{i}\right)=\mathrm{M}_{i}\right)$, but choose the following special ones:

$M_{4}$ equals $W, M_{2}$ coincides with I and $M_{3}$ with $J$. The corresponding platform anchor points are denoted by $m_{4}, m_{2}$ and $m_{3}$, respectively. As $M_{i}$ are ideal points the corresponding points $m_{i}$ are not running on spheres but in planes orthogonal to the direction of $\mathrm{M}_{i}$. Therefore these three point pairs imply three so-called Darboux conditions $\Omega_{i}$ for $i=2,3,4$. Moreover we denote $\mathrm{U}$ as $\mathrm{m}_{5}$ and its corresponding finite point under $\sigma$ by $M_{5}$. This point pair describes a so-called Mannheim condition $\Pi_{5}$ (which is the inverse of a Darboux condition). The pentapod is completed by a sphere condition $\Lambda_{1}$ of any pair of corresponding finite points $m_{1}$ and $M_{1}$.

In [1] we have chosen the fixed frame $\mathscr{F}_{0}$ in a way that $\mathrm{M}_{1}$ equals its origin and $\mathrm{M}_{4}$ coincides with the ideal point of the $z$-axis. Moreover we located the moving frame $\mathscr{F}$ in a way that $\mathrm{p}$ coincides with the $x$-axis, where $\mathrm{m}_{1}$ equals its origin.

For the study at hand it is advantageous to select a different set of fixed and moving frames $\mathscr{F}_{0}^{\prime}$ and $\mathscr{F}^{\prime}$, respectively:

- As $M_{2}$ and $M_{3}$ coincides with the cyclic points, we can assume without loss of generality (w.l.o.g.) that $\mathrm{M}_{5}$ is located in the $x z$-plane (as a rotation about the $\mathrm{z}$ axis does not change the coordinates of $\mathrm{M}_{1}, \ldots, \mathrm{M}_{4}$ ). Moreover we want to apply a translation in a way that $\mathrm{M}_{5}$ is in the origin of the new fixed frame $\mathscr{F}_{0}^{\prime}$. Summed up the coordinates with respect to $\mathscr{F}_{0}^{\prime}$ read as:

$$
\mathbf{M}_{5}=(0,0,0), \quad \mathbf{M}_{1}=(A, 0, C) \quad \text { with } \quad A \neq 0
$$

as $A=0$ implies a contradiction to the properties of $\mathrm{P}$ for Type 1 and Type 2 pentapods given in Section 1.1 Moreover, $\mathrm{M}_{2}, \mathrm{M}_{3}$ and $\mathrm{M}_{4}$ are the ideal points in direction $(1, i, 0)^{T},(1,-i, 0)^{T}$ and $(0,0,1)^{T}$, respectively.

\footnotetext{
${ }^{3}$ With respect to the notation introduced in Section 2 these five parameters are $C, a_{r}, a_{c}, a_{4}$ and $p_{5}$ or $R_{1}$ (cf. Eq. (7)) by canceling the factor of similarity by setting $A=1$.

${ }^{4}$ These are the parameters $a, c, g, k$ used in [10 Section 2.3].
} 
- With respect to $\mathscr{F}_{0}^{\prime}$ the location of $\mathrm{p}$ is undefined, but the coordinates $\mathbf{m}_{i}$ of $\mathrm{m}_{i}$ can be parametrized as follows for $i=1, \ldots, 4$ :

$$
\mathbf{m}_{i}=\mathbf{n}+\left(a_{i}-a_{r}\right) \mathbf{d} \quad \text { with } \quad a_{1}=0, a_{2}=a_{r}+i a_{c}, a_{3}=a_{r}-i a_{c}
$$

where $a_{r}, a_{c} \in \mathbb{R}$ and $a_{c} \neq 0$ holds. $\mathrm{m}_{5}$ is the ideal point in direction of the unitvector $\mathbf{d}=\left(d_{1}, d_{2}, d_{3}\right)^{T}$, which obtains the rational homogeneous parametrization of the unit-sphere, i.e.

$$
d_{1}=\frac{2 h_{0} h_{1}}{h_{0}^{2}+h_{1}^{2}+h_{2}^{2}}, \quad d_{2}=\frac{2 h_{0} h_{2}}{h_{0}^{2}+h_{1}^{2}+h_{2}^{2}}, \quad d_{3}=\frac{h_{1}^{2}+h_{2}^{2}-h_{0}^{2}}{h_{0}^{2}+h_{1}^{2}+h_{2}^{2}} .
$$

Now we are looking for the point $\mathbf{n}=\left(n_{1}, n_{2}, n_{3}\right)^{T}$ and the direction $\left(h_{0}: h_{1}: h_{2}\right)$ in a way that for the self-motion of the pentapod $e_{0}=f_{0}=0$ holds. We can discuss Type 1 and Type 2 at the same time, just having in mind that $a_{4} \neq 0 \neq C$ has to hold for Type 1 and $a_{4}=0=C$ for Type 2 (according to [1]).

By setting $\mathbf{r}_{i}:=\left(r_{i 1}, r_{i 2}, r_{i 3}\right)^{T}$ for $i=1,2,3$ the Darboux and Mannheim constraints with respect to $\mathscr{F}_{0}^{\prime}$ and $\mathscr{F}^{\prime}$ can be written as:

$$
\begin{array}{ll}
\Omega_{2}:\left(s_{1}+\mathbf{r}_{1} \mathbf{m}_{2}\right)-i\left(s_{2}+\mathbf{r}_{2} \mathbf{m}_{2}\right)-p_{2} N=0, & \Omega_{4}:\left(s_{3}+\mathbf{r}_{3} \mathbf{m}_{4}\right)-p_{4} N=0, \\
\Omega_{3}:\left(s_{1}+\mathbf{r}_{1} \mathbf{m}_{3}\right)+i\left(s_{2}+\mathbf{r}_{2} \mathbf{m}_{3}\right)-p_{3} N=0, & \Pi_{5}:(\mathbf{R d})\left(\mathbf{s}+\mathbf{R} \mathbf{p}_{5}\right) N^{-1}=0,
\end{array}
$$

with $\mathbf{p}_{5}=\mathbf{n}+\left(p_{5}-a_{r}\right) \mathbf{d}$, which is the coordinate vector of the intersection point of the Mannheim plane and $\mathrm{p}$ with respect to $\mathscr{F}^{\prime}$. Moreover $\left(p_{j}, 0,0\right)^{T}$ for $j=2,3$ (resp. $\left.\left(0,0, p_{4}\right)^{T}\right)$ are the coordinates of the intersection point of the Darboux plane and the $x$-axis (resp. $z$-axis) of $\mathscr{F}_{0}^{\prime}$.

Remark 1. As from the Mannheim constraint $\Pi_{5}$ of Eq. (5) the factor $N$ cancels out, all four constraints $\Omega_{2}, \Omega_{3}, \Omega_{4}, \Pi_{5}$ are homogeneous quadratic in the Study parameters and especially linear in $f_{0}, \ldots, f_{3}$.

According to [1, Theorems 13 and 14] the leg-parameters $p_{2}, \ldots, p_{5}, R_{1}$ have to fulfill the following necessary and sufficient conditions for the self-mobility (over $\mathbb{C})$ of a linear pentapod of Type 1 and Type 2, respectively:

$$
\begin{aligned}
& p_{2}=\frac{A a_{3} v}{\left(a_{3}-a_{4}\right)^{2}}, \quad p_{3}=\frac{A a_{2} v}{\left(a_{2}-a_{4}\right)^{2}}, \quad p_{4}=-\frac{C a_{4} v}{\left(a_{2}-a_{4}\right)\left(a_{3}-a_{4}\right)}, \\
& \left(a_{2}-a_{4}\right)^{2}\left(a_{3}-a_{4}\right)^{2}\left[2 w p_{5}-v R_{1}^{2}-\left(2 w-v a_{4}\right) a_{4}\right]+v w^{2}\left(A^{2}+C^{2}\right)=0,
\end{aligned}
$$

with $v:=a_{2}+a_{3}-2 a_{4}$ and $w:=a_{2} a_{3}-a_{4}^{2}$. Therefore if we set $p_{2}, p_{3}, p_{4}$ as given in Eq. (6) then only one condition in $p_{5}$ and $R_{1}$ remains in Eq. (7). Therefore these pentapods have a 1-dimensional set of self-motions.

Theorem 1. Each self-motion of a linear pentapod of Type 1 and Type 2 can be generated by a 1-dimensional set of line-symmetric motions. For the special case $p_{5}=a_{4}=a_{r}$ this set is even 2-dimensional. 
Proof. W.l.o.g. we can set $e_{0}=0$ as any two directions $\mathbf{d}$ of $\mathrm{p}$ can be transformed into each other by a half-turn about their enclosed bisecting line. Note that this line is not uniquely determined if and only if the two directions are antipodal.

W.l.o.g. we can solve $\Psi, \Omega_{2}, \Omega_{3}, \Omega_{4}$ for $f_{0}, f_{1}, f_{2}, f_{3}$ and plug the obtained expressions into $\Pi_{5}$, which yields in the numerator a homogeneous quartic polynomial $G[1563]$ in $e_{1}, e_{2}, e_{3}$, where the number in the brackets gives the number of terms. Moreover the numerator of the obtained expression for $f_{0}$ is denoted by $F[600]$, which is a homogeneous cubic polynomial in $e_{1}, e_{2}, e_{3}$.

General Case $(v \neq 0)$ : The condition $G=0$ already expresses the self-motion as $G$ equals $\Lambda_{1}$ if we solve Eq. (7) for $R_{1}$. Moreover $F=0$ has to hold if the self-motion of the line $\mathrm{p}$ can be generated by a line-symmetric motion. As for any solution $\left(e_{1}: e_{2}: e_{3}\right)$ of $F=0$ also $G=0$ has to hold, $G$ has to split into $F$ and a homogeneous linear factor $L$ in $e_{1}, e_{2}, e_{3}$.

Now $L=0$ cannot correspond to a self-motion of the linear pentapod, but has to arise from the ambiguity in representing a direction of $p$ mentioned at the beginning of the proof. This can be argued indirectly as follows:

Assumed $L=0$ implies a self-motion, then it has to be a Schönflies motion (with a certain direction $v$ of the rotation axis) due to $e_{0}=0$. As under such a motion the angle enclosed by $v$ and $p$ remains constan $[5$ the ideal point $U$ of $p$ has to be mapped by $\sigma$ to the ideal point $\mathrm{V}$ of $\mathrm{v}$. This implies that $\mathrm{V}$ has to coincide with $\mathrm{W}$, which can only be the case for pentapods of Type 5; a contradiction.

Therefore there has to exist a pose of $p$ during the self-motion, where it is oppositely oriented with respect to the fixed frame and moving frame, respectively. As a consequence we can set $L=d_{1} e_{1}+d_{2} e_{2}+d_{3} e_{3}$ which yields the ansatz:

$$
\Delta: \quad \lambda L F-G=0 .
$$

The resulting set of four equations arising from the coefficients of $e_{1}^{3} e_{2}, e_{1}^{3} e_{3}$, $e_{1} e_{3}^{3}$ and $e_{2} e_{3}^{3}$ of $\Delta$ has the unique solution:

$$
n_{1}=a_{c} d_{2}, \quad n_{2}=-a_{c} d_{1}, \quad n_{3}=\left(a_{r}-a_{4}\right) d_{3}, \quad \lambda=2\left(h_{0}^{2}+h_{1}^{2}+h_{2}^{2}\right) .
$$

Now $\Delta$ splits up into $\left(e_{1}^{2}+e_{2}^{2}+e_{3}^{2}\right)^{2}\left(h_{0}^{2}+h_{1}^{2}+h_{2}^{2}\right) H[177]$, where $H$ is homogeneous of degree 4 in $h_{0}, h_{1}, h_{2}$. For the explicit expression of the planar quartic curve $H=0$ see Remark 3, which is given right after this proof.

Remark 2. Note that all self-motions of the general case can be parametrized as the resultant of $G$ and the normalizing condition $N-1$ with respect to $e_{i}$ yields a polynomial, which is only quadratic in $e_{j}$ for pairwise distinct $i, j \in\{1,2\}$.

Special Case $(v=0)$ : If $v=0$ holds, we cannot solve Eq. (7) for $R_{1}$. The conditions $v=0$ and Eq. (7) imply $p_{5}=a_{4}=a_{r}$. Now $G$ is fulfilled identically and the selfmotion is given by $\Lambda_{1}=0$, which is of degree 4 in $e_{1}, e_{2}, e_{3}$. Moreover for this special case $F=0$ already holds for $\mathbf{n}$ given in Eq. 99. Therefore any direction $\left(h_{0}: h_{1}: h_{2}\right)$ for $\mathrm{p}$ can be chosen in order to fix the line-symmetric motion.

\footnotetext{
${ }^{5}$ This angle condition can be seen as the limit of the sphere condition (cf. [12, Section 4.1]).
} 

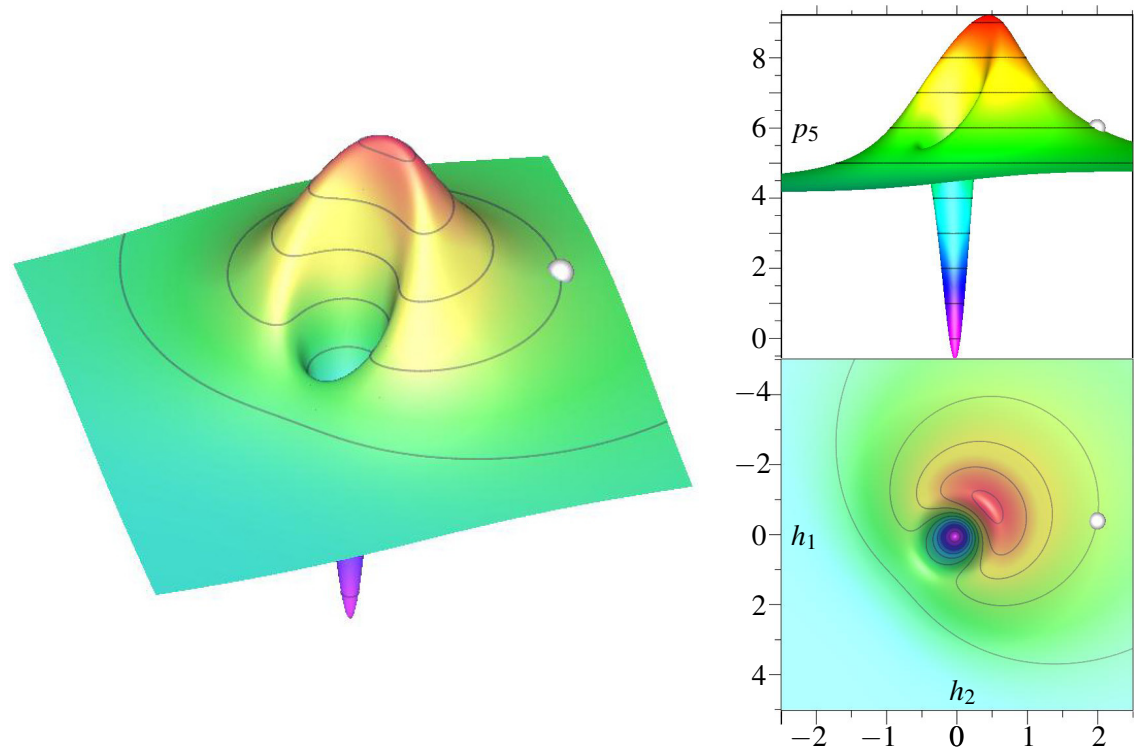

Fig. 2 For the example given in Eq. 13 the graph of $p_{5}$ in dependency of $h_{1}$ and $h_{2}$ with $h_{0}=1$ is displayed in the axonometric view on the left and in the front resp. top view on the right side. The highlighted point at height 6 corresponds to the values $h_{1}=-\frac{489262}{226525}+\frac{488}{226525} \sqrt{675091}$ and $h_{2}=\frac{535336}{226525}+\frac{446}{226525} \sqrt{675091}$.

Remark 3. $H=0$ represents a planar quartic curve, which can be verified to be entirely circular. Moreover $H=0$ can be solved linearly for $p_{5}$. The corresponding graph is illustrated in Fig. 2

If we reparametrize the $h_{0} h_{1} h_{2}$-plane in terms of homogenized polar coordinates by:

$$
h_{0}=\left(\tau_{1}^{2}+\tau_{0}^{2}\right) \rho_{0}, \quad h_{1}=\left(\tau_{1}^{2}-\tau_{0}^{2}\right) \rho_{1}, \quad h_{2}=2 \tau_{0} \tau_{1} \rho_{1},
$$

where $\left(\tau_{0}, \tau_{1}\right) \neq(0,0) \neq\left(\rho_{0}, \rho_{1}\right)$ and $\tau_{0}, \tau_{1}, \rho_{0}, \rho_{1} \in \mathbb{R}$ hold, then $H$ factors into $\left(\tau_{0}^{2}+\tau_{1}^{2}\right)^{3}\left(H_{2} \tau_{1}^{2}+H_{1} \tau_{0} \tau_{1}+H_{0} \tau_{0}^{2}\right)$ with

$$
\begin{aligned}
H_{1} & =8 \rho_{0} \rho_{1} A\left(a_{4}-a_{r}\right)\left(\rho_{1}^{2}+\rho_{0}^{2}\right)\left(a_{r}^{2}-a_{4}^{2}+a_{c}^{2}\right) a_{c}, \\
H_{0}-H_{2} & =8 \rho_{0} \rho_{1} A\left(a_{4}-a_{r}\right)\left(\rho_{1}^{2}+\rho_{0}^{2}\right)\left[a_{r}\left(a_{r}-a_{4}\right)^{2}+a_{c}^{2}\left(a_{r}-2 a_{4}\right)\right], \\
H_{0}+H_{2} & =2\left[\left(a_{r}-a_{4}\right)^{2}+a_{c}^{2}\right]\left[2 a_{4}\left(\rho_{1}^{4}-\rho_{0}^{4}\right)\left(a_{4}-a_{r}\right) C+\right. \\
& \left.\left(\left(a_{r}-a_{4}\right)^{2}+a_{c}^{2}\right)\left(\left(\rho_{0}^{4}+\rho_{1}^{4}\right)\left(a_{4}-p_{5}\right)+2 \rho_{0}^{2} \rho_{1}^{2}\left(2 a_{r}-a_{4}-p_{5}\right)\right)\right] .
\end{aligned}
$$

Therefore this equation can be solved quadratically for the homogeneous parameter $\tau_{0}: \tau_{1}$. Note that the value $p_{5}$ is fixed during a self-motion. 


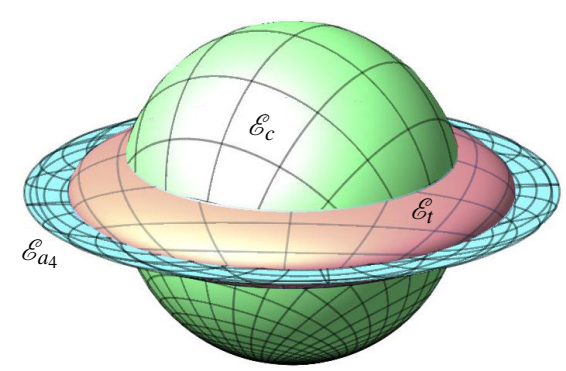

(a)

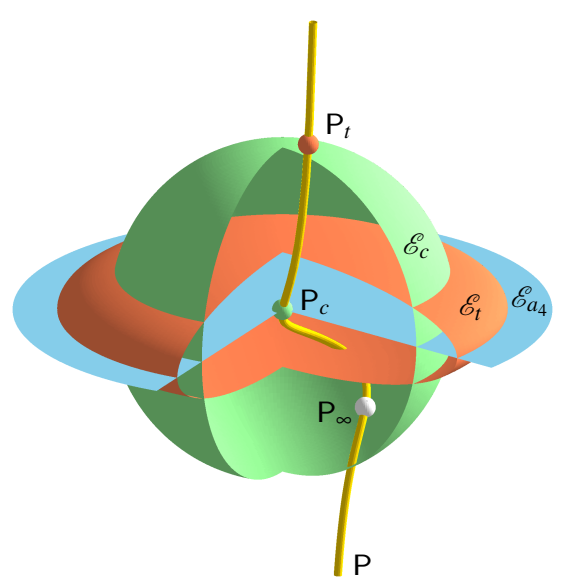

(b)

Fig. 3 For the example given in Eq. 13 the loci $\mathscr{E}_{a_{4}}, \mathscr{E}_{c}$ and $\mathscr{E}_{t}$ with $t=\frac{69}{20}$ are illustrated. (a) For $h_{0}=1$ the $h_{1}$ - and $h_{2}$-parameter lines of the given rational quadratic parametrization are displayed. (b) The loci are sliced (along the not drawn axis of rotation c) in order to visualize their positioning with respect to the cubic $\mathrm{P}$ on which the points $\mathrm{P}_{\infty}=\sigma(\mathrm{U}), \mathrm{P}_{c}=\mathrm{C}$ and $\mathrm{P}_{t}$ are highlighted. Note that $\mathrm{P}_{a_{4}}=\mathrm{W}$ is the real ideal point of $\mathrm{P}$.

\section{On the reality of Type 1 and Type 2 self-motions}

A similar computation to [1, Example 1] shows that for any real point $\mathrm{p}_{t} \in \mathrm{p}$ with $t \in \mathbb{R}$ and coordinate vector $\mathbf{p}_{t}=\mathbf{n}+\left(t-a_{r}\right) \mathbf{d}$ with respect to $\mathscr{F}^{\prime}$ the corresponding real point $\mathrm{P}_{t} \in \mathrm{P}$ has the following coordinate vector $\mathbf{P}_{t}$ with respect to $\mathscr{F}_{0}^{\prime}$ :

$$
\mathbf{P}_{t}=\left(\frac{A\left(a_{r}^{2}+a_{c}^{2}-t a_{r}\right)}{\left(t-a_{r}\right)^{2}+a_{c}^{2}},-\frac{A a_{c} t}{\left(t-a_{r}\right)^{2}+a_{c}^{2}}, \frac{C a_{4}}{a_{4}-t}\right)^{T} .
$$

As $L=0$ corresponds with one configuration of the self-motion we can compute the locus $\mathscr{E}_{t}$ of $\mathrm{p}_{t}$ with respect to $\mathscr{F}_{0}^{\prime}$ under the 1-parametric set of self-motions by the variation of $\left(h_{0}: h_{1}: h_{2}\right)$ within $L=0$. Moreover due to the mentioned ambiguity we can select an arbitrary solution $\left(e_{0}: e_{1}: e_{2}\right)$ for $L=0$ fulfilling the normalization condition $N=1$; e.g.: $e_{1}=h_{2}\left(h_{1}^{2}+h_{2}^{2}\right)^{-\frac{1}{2}}, e_{2}=-h_{1}\left(h_{1}^{2}+h_{2}^{2}\right)^{-\frac{1}{2}}$ and $e_{3}=0$. Now the computation of $\mathbf{R} \mathbf{p}_{t}+\mathbf{s}$ yields a rational quadratic parametrization of $\mathscr{E}_{t}$ (see Fig. 3 a) in dependency of $\left(h_{0}: h_{1}: h_{2}\right)$.

Note that this approach also includes the special case $(v=0)$ as there always exists a value for $R_{1}^{2}$ (in dependency of $\left(h_{0}: h_{1}: h_{2}\right)$ ) in a way that $\Lambda_{1}=0$ holds.

For $t \neq a_{4}$ all $\mathscr{E}_{t}$ are ellipsoids of rotation, which have the same center point $C$ and axis of rotation $\mathrm{C}$ (see Fig. 3b). In detail, C is the point of the straight cubic circle 12 for the value $t=c$ with $c:=\frac{a_{4}^{2}-a_{c}^{2}-a_{r}^{2}}{2\left(a_{4}-a_{r}\right)}$ (for $a_{4}=a_{r}$ we get $c=\infty$ thus $\mathrm{p}_{\infty}=\mathrm{U}=\mathrm{m}_{5}$ holds, which implies $\mathrm{C}=\mathrm{M}_{5}$ ) and $\mathrm{c}$ is parallel to the $z$-axis of $\mathscr{F}_{0}^{\prime}$. 


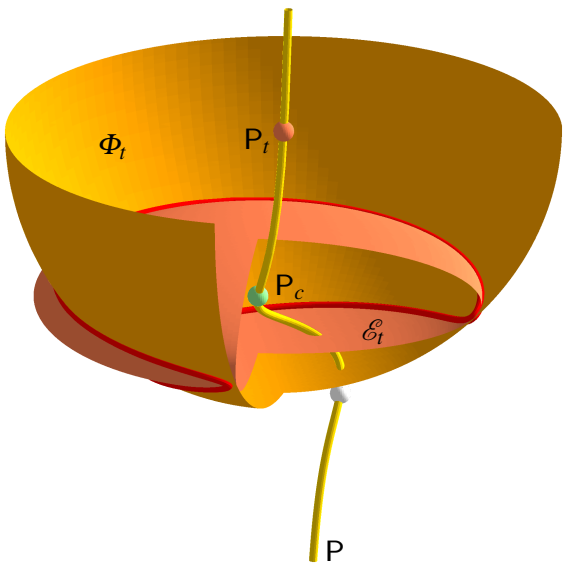

(a)

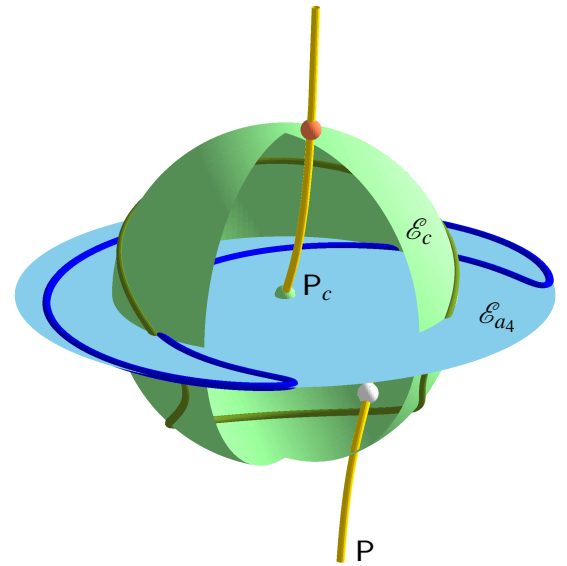

(b)

Fig. 4 For the example given in Eq. 13 and $p_{5}=6$ the trajectory of $\mathrm{p}_{t}$ with $t=\frac{69}{20}$ is illustrated in (a) as the intersection curve of $\mathscr{E}_{t}$ and the sphere $\Phi_{t}$ centered in $\mathrm{P}_{t}$. The trajectories of the points $\mathrm{p}_{c}$ and $\mathrm{p}_{a_{4}}$, where this intersection procedure for their generation fails, are visualized in (b) as curves on $\mathscr{E}_{c}$ and $\mathscr{E}_{a_{4}}$, respectively.

Moreover the vertices on $c$ have distance $\left|a_{4}-t\right|$ from $C$ and the squared radius of the equator circle equals $\left(a_{r}-t\right)^{2}+a_{c}^{2}$. Note that for $a_{4} \neq a_{r}$ the only sphere within the described set of ellipsoids is $\mathscr{E}_{c}$. For $a_{4}=a_{r}$ no such sphere exists.

$\mathscr{E}_{a_{4}}$ is a circular disc in the Darboux plane $z=p_{4}$ (w.r.t. $\mathscr{F}_{0}^{\prime}$ ) centered in C.

Remark 4. The existence of these ellipsoids was already known to Duporcq [8, §9], who used them to show that the spherical trajectories are algebraic curves of degree 4 (see Fig. 4).

Based on this geometric property, recovered by line-symmetric motions, we can formulate the condition for the self-motion to be real as follows:

- $w \neq 0$ : We can reduce the problem to a planar one by intersecting the plane spanned by $\mathrm{P}_{0}=\mathrm{M}_{1}$ and $\mathrm{c}$ with $\mathscr{E}_{0}$ and the sphere with radius $R_{1}$ centered in $\mathrm{P}_{0}$. Now there exists an interval $\left.I_{0}=\right] I_{-}, I_{+}\left[\right.$such that for $R_{1} \in I_{0}$ the two resulting conics have at least two distinct real intersection points. It is well known (e.g. [13]) that the computation of the limits $I_{-}$and $I_{+}$of the reality interval $I_{0}$ leads across an algebraic problem of degree 4 (explicitly solvable). Thus for a real self-motion we have to choose $R_{1} \in I_{0}$ and solve Eq. (7) for $p_{5}$.

- $w=0$ : Now $\mathrm{P}_{0}$ coincides with $\mathrm{C}$ and the interval collapses to the single value $R_{1}=\left|a_{4}\right|$, which can be seen from Eq. (7). Moreover $p_{5}$ can be chosen arbitrarily.

These considerations also show that any pentapod of Type 1 and 2 has real selfmotions if the leg-parameters are chosen properly. Note that this is e.g. not the case for some designs of Type 5 pentapods described in [1, Section 6], where it was also 


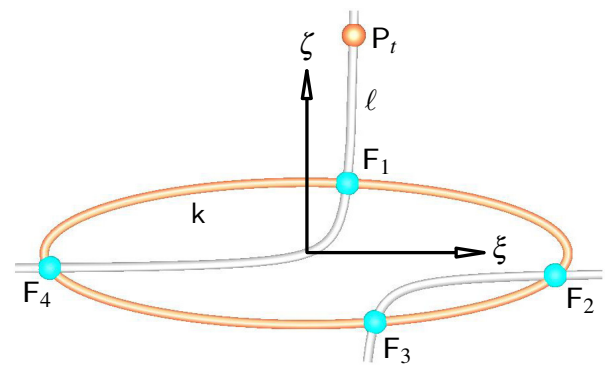

Fig. 5 Lagrange curve $\ell$ intersects the ellipse $\mathrm{k}$ in the pedal points $\mathrm{F}_{i}(i=1, \ldots, 4)$ of $\mathrm{k}$ w.r.t. $\mathrm{P}_{t}$.

proven that pentapods with self-motions have a quartically solvable direct kinematics. It is possible to use this advantage (closed form solution) of pentapods with self-motions without any risk 6 , by designing linear pentapods of Type 1 and Type 2, which are guaranteed free of self-motions within their workspace.

A sufficient condition for that is that (at least) for one of the five legs $p_{t} P_{t}$ of the pentapod the corresponding reality interval $I_{t}$ is disjoint with the interval of the maximal and minimal leg length implied by the mechanical realization. This condition for a self-motion free workspace gets especially simple if $\mathrm{p}_{c} \mathrm{P}_{c}$ is this leg.

Example 1. We only provide an example for the most general case; i.e. Type 1 pentapod with self-motion. The parameters are chosen as follows:

$$
a_{4}=2, \quad A=-1, \quad C=-5, \quad a_{r}=7, \quad a_{c}=4,
$$

with respect to the frames $\mathscr{F}_{0}^{\prime}$ and $\mathscr{F}^{\prime}$, respectively. In Fig. 5 the planar intersection of $\mathscr{E}_{t}$ for $t=\frac{69}{20}$ with the plane spanned by $\mathrm{P}_{t}$ and $\mathrm{c}$ is illustrated. The half-axes lengths of this ellipse $\mathrm{k}$ are $k_{1}=\frac{\sqrt{11441}}{20}$ and $k_{2}=\frac{29}{20}$.

With respect to a planar Cartesian frame $(\xi, \zeta)$ aligned with the axes of the ellipse $\mathrm{k}$ the point $\mathrm{P}_{t}$ has the coordinates $\left(\xi_{t}, \zeta_{t}\right):=\left(\frac{530}{469081} \sqrt{743665}, \frac{5300}{1189}\right)$. Then the socalled Lagrange curve $\ell$, which intersects $k$ in the pedal points with respect to $P_{t}$, has the following parametrization (parameter $\mathrm{k}$ ) with respect to the planar Cartesian frame $(\xi, \zeta)$ :

$$
\left(k, \frac{\kappa_{1} \zeta_{t} k}{\left(\kappa_{1}-\kappa_{2}\right) k+\kappa_{2} \xi_{t}}\right) \quad \text { with } \quad \kappa_{1}=\frac{1}{k_{1}^{2}} \quad \text { and } \quad \kappa_{2}=\frac{1}{k_{2}^{2}} .
$$

Plugging this parametrization into the equation of the ellipse k, given by $\kappa_{1} \xi^{2}+$ $\kappa_{2} \zeta^{2}=1$ yields a quartic equation. Therefore the pedal points $F_{i}$ for $i=1, \ldots 4$ and the corresponding distances $l_{i}$ to $\mathrm{P}_{t}$ can be computed explicitly. Then $I_{-}$and $I_{+}$of $I_{t}$ are given by the minimal and maximal value of $\left\{l_{1}, l_{2}, l_{3}, l_{4}\right\}$. For the example under consideration, the corresponding rounded numerical values are $I_{-} \approx 3.02850$ and $I_{+} \approx 7.82039$, respectively.

\footnotetext{
6 A self-motion is dangerous as it is uncontrollable and thus a hazard to man and machine.
} 


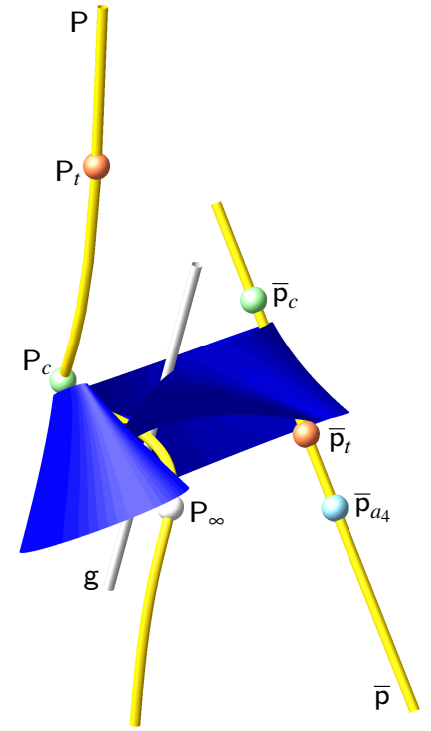

(a)

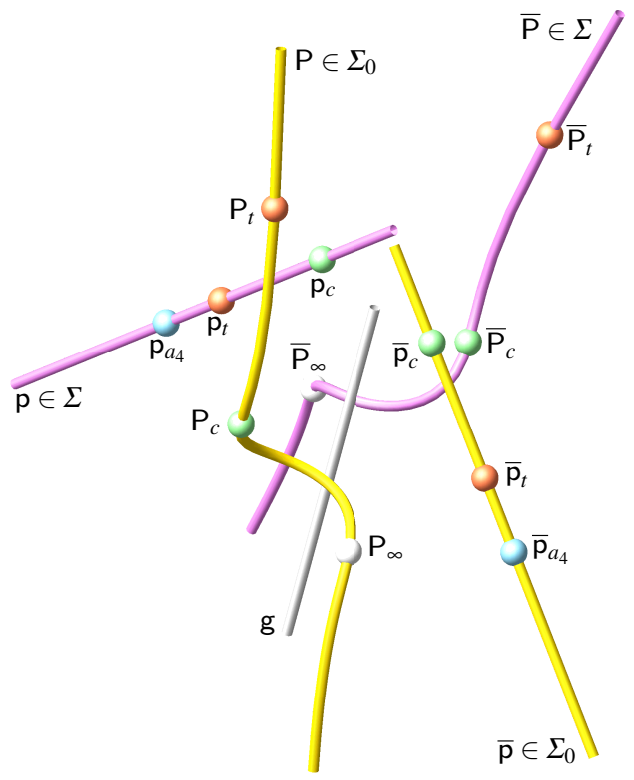

(b)

Fig. 6 (a) For the example given in Eq. 13 the basic surface is illustrated for the parameter values highlighted in Fig. 2 In addition $P$ and $\bar{p}$ are visualized, where the latter denotes the pose of $p$ such that its half-turns about the generators of the basic surface yield the self-motion. (b) The construction outlined by Krames [5, page 416] is illustrated with respect to the generator $g$ of the basic surface: As $\mathrm{P}_{a_{4}}$ (resp. $\overline{\mathrm{p}}_{\infty}$ ) is the real ideal point of $\mathrm{P}$ (resp. $\overline{\mathrm{p}}$ ), the trajectory of $\mathrm{p}_{a_{4}}$ (resp. $\overline{\mathrm{P}}_{\infty}$ ) under the self-motion $\mu$ is planar. The (Mannheim) plane $\in \Sigma$, which contains the point $\mathrm{P}_{\infty}$ (resp. $\overline{\mathrm{p}}_{a_{4}}$ ) and is orthogonal to the direction of the real ideal point $\mathrm{p}_{\infty}\left(\right.$ resp. $\overline{\mathrm{P}}_{a_{4}}$ ) of $\mathrm{p}($ resp. $\overline{\mathrm{P}}$ ) in the displayed pose, slides through the point $\mathrm{P}_{\infty}\left(\right.$ resp. $\left.\overline{\mathrm{p}}_{a_{4}}\right)$ during the complete motion $\mu$.

Remark 5. A straight forward computation for the general case (cf. example given in the Appendix) shows that the basic surface is of degree 5 (see Fig. 6a). Moreover the intersection of this basic surface with the ideal plane decomposes into a cubic curve and the two tangents $(0: 1: \pm i: \mathbb{R})$ to the absolute circle. According to Krames [14, Theorem 8 and the subsequent paragraph] a general point of the moving system $\Sigma$ has a trajectory of degree 6 under the corresponding line-symmetric motion (see Fig. 77.

Note that these quintic basic surfaces differ from those studied by Krames in [15], as during the corresponding line-symmetric motions of the latter no point of $\Sigma$ can have a spherical path due to the following reason: Krames showed in [15, page 230] that every point has a trajectory of degree 4. Due to [5, Theorem 7] the existence of points with spherical trajectories already implies that the basic surface has to be of degree 4; a contradiction. 


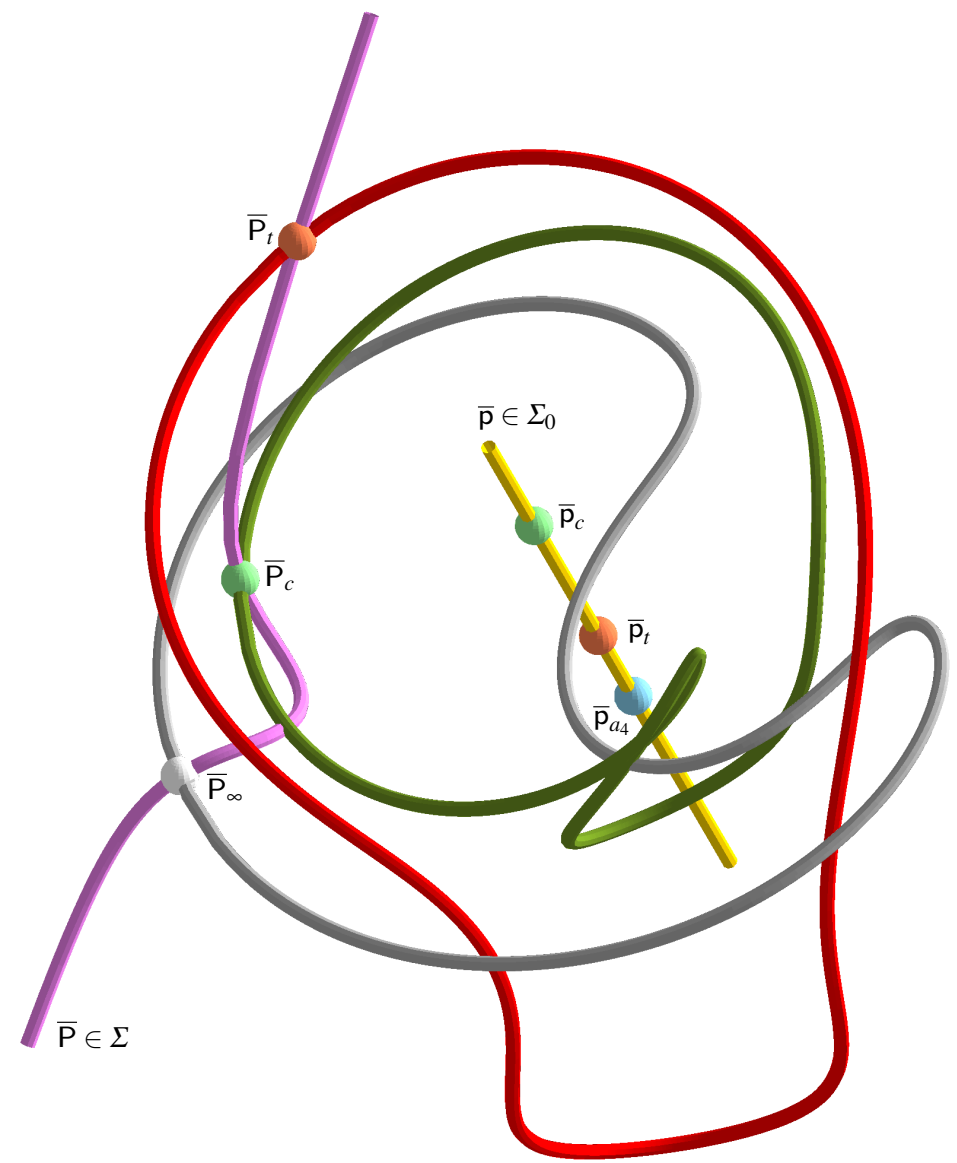

Fig. 7 Also the points of the cubic $\bar{P} \in \Sigma$ have trajectories of degree 6 under the corresponding line-symmetric motion. The paths of $\overline{\mathrm{P}}_{\infty}$ (gray), $\overline{\mathrm{P}}_{t}$ (red) and $\overline{\mathrm{P}}_{c}$ (green) are illustrated for the linesymmetric motion implied by the basic surface displayed in Fig. 6. Note that in contrast to the Figs. 2 3 4 and 6 the camera location was changed as otherwise the planar trajectory of $\overline{\mathrm{P}}_{\infty}$ would have been collapsed into a line-segment.

\section{Conclusion and open problem}

Krames [5, page 416] outlined the following construction illustrated in Fig. 6p: Assume that $\mathrm{p}$ is in an arbitrary pose of the self-motion $\mu$ with respect to $\mathrm{P}$, where $\mathrm{g}$ denotes the generator of the basic surface, which corresponds to this pose. Moreover $\bar{p}$ and $\bar{P}$ are obtained by the reflexion of $p$ and $P$, respectively, with respect to $g$, where $\overline{\mathrm{p}}$ belongs to the fixed system $\Sigma_{0}$ and $\overline{\mathrm{P}}$ to the moving system $\Sigma$. Then under the self-motion $\mu$ also the points of $\overline{\mathrm{P}}$ are located on spheres with centers on the line $\bar{p}$. 
We can apply this construction for each line-symmetric motion of Theorem 1 . which yields new solutions for the Borel Bricard problem, with the exception of one special case where $\mathrm{W} \in \overline{\mathrm{p}}$ holds (i.e. $h_{1}=h_{2}=0$ or $h_{0}=0$ ), which was already given by Borel in [2, Case Fa4]. Moreover for this case Borel noted that beside $p$ and $\bar{P}$ only two imaginary planar cubic curves, which are located in the isotropic planes through $p$, run on spheres. The example given in the Appendix shows that this also holds true for the general case.

Thus the problem remains to determine all line-symmetric motions of Theorem 1 where additional real points (beside those of $p$ and $\bar{P}$ ) run on spheres. Until now the only examples with this property, which are known to the author, are the BB-II motions (cf. Section 1.3 .

Acknowledgements This research is funded by Grant No. P 24927-N25 of the Austrian Science Fund FWF within the project Stewart Gough platforms with self-motions.

\section{References}

1. Nawratil, G., Schicho, J.: Self-motions of pentapods with linear platform. Robotica, accepted |arXiv:1407.6126]

2. Borel, E.: Mémoire sur les déplacements à trajectoires sphériques. Mémoire présenteés par divers savants à l'Académie des Sciences de l'Institut National de France 33(1) 1-128 (1908)

3. Bricard, R.: Mémoire sur les déplacements à trajectoires sphériques. Journal de École Polytechnique(2) 11 1-96 (1906)

4. Husty, M.: E. Borel's and R. Bricard's Papers on Displacements with Spherical Paths and their Relevance to Self-Motions of Parallel Manipulators. International Symposium on History of Machines and Mechanisms (M. Ceccarelli ed.), 163-172, Kluwer (2000)

5. Krames, J.: Zur Bricardschen Bewegung, deren sämtliche Bahnkurven auf Kugeln liegen. Monatsheft für Mathematik und Physik 45 407-417 (1937)

6. Koenigs, G.: Leçons de Cinématique (avec notes par G. Darboux). Paris (1897)

7. Mannheim, A.: Principes et Développements de Géométrie Cinématique. Paris (1894)

8. Duporcq, E.: Sur le déplacement le plus général d'une droite dont tous les points décrivent des trajectoires sphériques. Journal de mathématiques pures et appliquées (5) 4 121-136 (1898)

9. Bottema, O., Roth, B.: Theoretical Kinematics. North-Holland Publishing Company (1979)

10. Hartmann, D.: Singular Stewart-Gough Platforms. Master Thesis, Department of Mechanical Engineering, McGill University, Montreal, Canada (1995)

11. Krames, J.: Die Borel-Bricard-Bewegung mit punktweise gekoppelten orthogonalen Hyperboloiden. Monatsheft für Mathematik und Physik 46 172-195 (1937)

12. Nawratil, G.: Correcting Duporcq's theorem. Mechanism and Machine Theory 73 282-295 (2014)

13. Chernova, N., Wijewickrema, S.: Algorithms for projecting points onto conics. Journal of Computational and Applied Mathematics 251 8-21 (2013)

14. Krames, J.: Über Fußpunktkurven von Regelflächen und eine besondere Klasse von Raumbewegungen. Monatshefte für Mathematik und Physik 45 394-406 (1936)

15. Krames, J.: Über eine konoidale Regelfläche fünften Grades und die darauf gegründete symmetrische Schrotung. Sitzungsbericht der Österreichischen Akademie der Wissenschaften Mahem.-naturw. Klasse, Abteilung II, 190(4-7) 221-230 (1981)

16. Husty, M.L.: An algorithm for solving the direct kinematics of general Stewart-Gough platforms. Mechanism and Machine Theory 31(4) 365-380 (1996) 


\section{Appendix}

We continue the example given in Eq. 13) for the parameters

$$
h_{0}=1, \quad h_{1}=\frac{3}{2}, \quad h_{2}=\frac{1}{2} .
$$

Now $H=0$ implies the condition $p_{5}=\frac{527538}{82369}$.

\section{Degree of basic surface}

As already mentioned at the end of Section 1.2, $\left(e_{1}: e_{2}: e_{3}: f_{1}: f_{2}: f_{3}\right)$ are the Plücker coordinates of the generators of the basic surface with respect to the fixed frame $\mathscr{F}_{0}^{\prime}$ if $e_{0}=f_{0}=0$ holds. Each generator $\mathrm{g}$ can now be parametrized by

$$
\mathrm{g}: \mathbf{G}+\gamma \mathbf{e} \text { with } \mathbf{G}:=\mathbf{f} \times \mathbf{e}
$$

where $\mathbf{e}:=\left(e_{1}, e_{2}, e_{3}\right)^{T}$ and $\mathbf{f}:=\left(f_{1}, f_{2}, f_{3}\right)^{T}$. Note that $\mathbf{G}$ is the coordinate vector (with respect to $\mathscr{F}_{0}^{\prime}$ ) of the pedal point of $\mathrm{g}$ with respect to the origin of $\mathscr{F}_{0}^{\prime}$. As $f_{0}, \ldots, f_{3}$ are computed as in Theorem 1, the parametrization of points of the basic surface, which have fixed coordinates $(X, Y, Z)^{T}$, only depends on $e_{1}, e_{2}, e_{3}$ and $\gamma$. Moreover the condition $F=0$ (implied by $f_{0}=0$ ) has to hold with:

$$
\begin{aligned}
F:= & 1561 e_{1}^{3}-1708 e_{2}^{3}-2870 e_{3}^{3}-1708 e_{1}^{2} e_{2}+2173 e_{1}^{2} e_{3} \\
& +1561 e_{1} e_{2}^{2}+2173 e_{2}^{2} e_{3}-8525 e_{1} e_{3}^{2}-5070 e_{2} e_{3}^{2} .
\end{aligned}
$$

From $F=0$ and the three equations $(X, Y, Z)^{T}=\mathbf{G}+\gamma \mathbf{e}$ the parameters $e_{1}, e_{2}, e_{3}$ and $\gamma$ can be eliminated (by Gröbner basis elimination techniques) and we finally end up with the following implicit representation of the basic surface with respect to $\mathscr{F}_{0}^{\prime}$ :

$$
\begin{aligned}
& 46930000-59658690 X^{2} Y Z^{2}-187188000 X-211012100 Z-214586000 Y \\
& -100313675 X Y^{2} Z^{2}+51139382 X^{2} Y^{2} Z+199127898 X^{2} Y Z-109272380 X Y Z^{2} \\
& +507256879 X Y^{2} Z+812997056 X Y Z+18368287 X^{5}+156741893 X^{4}+193802233 X^{3} \\
& -138195885 X^{2}+323499460 Y^{2}-423262296 Y^{3}-191807553 Y^{4}-20098036 Y^{5} \\
& +115381175 Z^{2}-53539850 Z^{3}-20098036 X^{4} Y+36736574 X^{3} Y^{2}-40196072 X^{2} Y^{3} \\
& +18368287 X Y^{4}-5063828 X^{3} Y-35065660 X^{2} Y^{2}-5063828 X Y^{3}+378103208 X^{2} Y \\
& -582502914 X Y^{2}+195340960 X Y+25569691 X^{4} Z-100313675 X^{3} Z^{2}-33771290 X^{2} Z^{3} \\
& +507256879 X^{3} Z-176781955 X^{2} Z^{2}-84016380 X Z^{3}+1163441469 X^{2} Z+34883415 X Z^{2} \\
& +556424125 X Z+13179040 Y Z^{3}+639976950 Y Z-33771290 Y^{2} Z^{3}-28318339 Y^{2} Z \\
& +199127898 Y^{3} Z-59658690 Y^{3} Z^{2}+25569691 Y^{4} Z+96060335 Y^{2} Z^{2}-41015170 Y Z^{2}=0 .
\end{aligned}
$$


It can easily be seen that this is a polynomial equation of degree 5. If we intersect this quintic surface with the ideal plane (by homogenizing it with the homogenization variable $W$ and then setting $W=0$ ) the intersection curve splits up into the two tangents to the absolute circle $X \pm Y i=0$ and the cubic curve given by the direction vectors of the basic surface's generators. Therefore this cubic curve in the ideal plane is given by $F=0$ (with $F$ of Eq. (17)) if one substitutes $e_{1}$ by $X, e_{2}$ by $Y$ and $e_{3}$ by $Z$, respectively.

As the ideal curve of the basic surface contains two tangents of the absolute circle, a general point of the moving system $\Sigma$ has a trajectory of degree 6 under the corresponding line-symmetric motion according to Krames [14, Theorem 8 and the subsequent paragraph].

Remark 6. It should be mentioned that all basic surfaces and trajectories can be parametrized for the general case due to Remark 2, which was used for the generation of Figs. 6a and 7, respectively.

\section{Imaginary planar cubic curves}

Now we determine the set of all points $\in \Sigma$, which are running on spheres during the above given line-symmetric motion.

By using the Study parametrization of Euclidean displacements, the condition that a point of $\Sigma$ with coordinates $(x, y, z)^{T}$ (with respect to the moving frame $\left.\mathscr{F}^{\prime}\right)$ is located on a sphere centered in a point with coordinates $(X, Y, Z)^{T}$ (with respect to $\left.\mathscr{F}_{0}^{\prime}\right)$ is a quadratic homogeneous equation according to Husty [16]. For $e_{0}=0$ this so-called sphere condition $\Lambda=0$ reads as:

$$
\begin{aligned}
\Lambda:= & \left(x^{2}+y^{2}+z^{2}+X^{2}+Y^{2}+Z^{2}-R^{2}\right)\left(e_{1}^{2}+e_{2}^{2}+e_{3}^{2}\right)+4\left(f_{0}^{2}+f_{1}^{2}+f_{2}^{2}+f_{3}^{2}\right) \\
& -2(x X-y Y-z Z) e_{1}^{2}+2(x X-y Y+z Z) e_{2}^{2}+2(x X+y Y-z Z) e_{3}^{2} \\
& -4(y X+x Y) e_{1} e_{2}-4(z X+x Z) e_{1} e_{3}-4(z Y+y Z) e_{2} e_{3} \\
& -4(x+X)\left(e_{3} f_{2}-e_{2} f_{3}\right)-4(y+Y)\left(e_{1} f_{3}-e_{3} f_{1}\right)-4(z+Z)\left(e_{2} f_{1}-e_{1} f_{2}\right) \\
& +4(x-X) e_{1} f_{0}+4(y-Y) e_{2} f_{0}+4(z-Z) e_{3} f_{0},
\end{aligned}
$$

where $R$ denotes the radius of the sphere.

The corresponding Maple Worksheet of the following computations can be downloaded as mws file and pdf file from the links provided in the footnote $7 \mathrm{We}$ compute $f_{0}, \ldots, f_{3}$ as in Theorem 1 and plug them into $\Lambda$. The numerator of the resulting expression is denoted by $\Gamma[161]$, which is of degree 4 in $e_{1}, e_{2}, e_{3}$. Therefore we can make an ansatz of the form:

$$
F\left(\eta_{1} e_{1}+\eta_{2} e_{2}+\eta_{3} e_{3}\right)-\Gamma=0
$$

\footnotetext{
7 The links are http://www.dmg.tuwien.ac.at/nawratil/linearpentapod.mws and http://www.dmg.tuwien.ac.at/nawratil/linearpentapod.pdf, respectively.
} 
As this equation has to be fulfilled identically for all $e_{1}, e_{2}, e_{3}$, the coefficients with respect to these variables have to vanish. This results in a set of 15 equations from which we eliminate (by Gröbner basis elimination techniques) the unknowns $\eta_{1}, \eta_{2}, \eta_{3}, X, Y, Z, R$. We finally end up with an ideal $I$ in $x, y, z$ of degree 10 and dimension 1; i.e. there exists a curve of degree 10 , those points run on spheres during the line-symmetric motion.

This curve of degree 10 has to split in at least three components as we already know of the existence of a linear and a cubic component, namely $p$ and $\bar{P}$, respectively. Now we show that the remaining sextic splits up into conjugate complex planar cubics, which are located in the isotropic planes $\varepsilon_{1}, \varepsilon_{2} \in \Sigma$ through $\mathrm{p}$. These isotropic planes are given by:

$$
\varepsilon_{1,2}: \quad 91 x-84 y-126 z-122 \pm(147 y-98 z+714) i=0,
$$

with respect to $\mathscr{F}^{\prime}$. We express $x$ from it and plug it into the generating polynomials of $I$. It can easily be checked that the greatest common divisor of all resulting expression equals $(102+21 y-14 z)(\Re \pm \Im i)$ with

$$
\begin{aligned}
\Re:= & 274400 y^{3}+3374238 y z+13169366 z+3927840 y^{2}-30870 y^{2} z \\
& -5472908 z^{2}-1165514 y z^{2}+15910300 y+113190 z^{3}+17761620 \\
\mathfrak{I}:= & 984410 y^{2} z-1840195 y^{2}+9573816 y z-115248 y z^{2}-15809850 y \\
& -29479660+817369 z^{2}+20061237 z-408170 z^{3}
\end{aligned}
$$

The condition $102+21 y-14 z=0$ yields the line $\mathrm{p}$ and $\mathfrak{R} \pm \mathfrak{I} i=0$ determines the imaginary planar cubic curves. This result closes the Appendix. 